\title{
Grabados paleolíticos en la Cueva del Tajo de las Figuras (Benalup, Cádiz)
}

\author{
SERGIO RIPOLL LOPEZ * \\ MARTI MAS CORNELLA ** \\ Guadalupe Torra COLELL ***
}

Durante la campaña de 1991 del proyecto de investigación Las manifestaciones rupestres prehistóricas de la zona gaditana, actividades arqueológicas autorizadas y subvencionadas por la Dirección General de Bienes Culturales de la Consejería de Cultura y Medio Ambiente de la Junta de Andalucía y dirigidas por M. Mas, se nos encargó, en el mes de octubre, la excavación de urgencia de dos yacimientos de Sierra Momia que podian correr peligro inminente de destrucción. El primero de ellos - Cuevas de Levante- fue objeto de un sondeo estratigráfico, pudiéndose comprobar que contenía un material, que a falta de otros datos, atribuimos provisionalmente a un Solutrense Superior Evolucionado o bien a un Magdaleniense Inicial. En el otro yacimiento - Cubeta de la Paja-, situado a escasos metros del Tajo de las Figuras, también intentamos realizar un sondeo, pero, por la morfología de la estación, pudimos comprobar que el relleno era prácticamente inexistente y además estaba totalmente revuelto.

Con motivo de esta excavación realizamos una visita a la Cueva del Tajo de las Figuras (fig. 1). Al observar nuevamente los paneles pintados

* Profesor Titular del Departamento de Prehistoria e Historia Antigua de la UNED.

* Becario del Departamento de Prehistoria e Historia Antigua de la UNED.

*** Licenciada en Geografia e Historia, colaboradora del proyecto de investigación Las manifestaciones rupestres prehistóricas de la zona gaditana. 
nos dimos cuenta de la existencia de un gran número de grabados que habian pasado desapercibidos en anteriores investigaciones, al ser confundidos, debido a la espesa capa de alteración que recubre las paredes rocosas, con líneas creadas por planos de estratificación o trazos más o menos recientes. La amplia experiencia en el estudio del arte rupestre paleolítico de uno de nosotros hizo que siguiéramos algunas de estas líneas, comprobando que se trataba de representaciones claramente paleolíticas.

La Cueva del Tajo de las Figuras ya era conocida desde antiguo por los cabreros que habitaban en las proximidades, pero la primera noticia cientifica de la existencia de pinturas procede de $\mathrm{V}$. Molina, quien en abril de 1913 visitó la cavidad y redactó un breve informe sobre su viaje (Mo. LINA, V., 1913). Este escrito hizo que J. Cabré y E. Hernández-Pacheco se desplazaran en junio de este mismo año a Cádiz para comprobar in situ este descubrimiento, reanudando sus trabajos de campo en abril y mayo de 1914 y hallando otras estaciones con arte rupestre (HERNANDEZ Pacheco, E., y Cabré, J., 1913; Cabré, J., y Hernandez Pacheco, E., 1914). Entre diciembre de 1913 y marzo de 1914, H. Breuil, acompañado por $W$. Verner, quien ya conocía la cavidad por sus expediciones ornitológicas, estuvo en la zona estudiando estos hallazgos, que le llevarían a publicar, junto con M. C. Burkitt, después de haber reanudado las investigaciones en 1916, 1918 y 1919, su conocida obra, en la que dedica un amplio apartado a la Cueva del Tajo de las Figuras y describe los restantes lugares conocidos de las sierras que bordeaban la laguna de la Janda, hoy desecada (Breult, H., y BurkitT, M. C., 1929).

Para J. Cabré y E. Hernández-Pacheco las pinturas de la Cueva del Tajo de las Figuras pueden englobarse en tres grandes grupos: figuras humanas, representaciones de animales y signos. Apreciando el distinto grado de estilización, basándose en la suposición de que cuanto más patente sea ésta más modernas son las figuras, y teniendo en cuenta las superposiciones, establecen una secuencia que situa las pinturas rojas y amarillas entre las más antiguas, mientras que las rojas de color intenso serían posteriores y las blancas más recientes. A partir del estudio estilístico y comparaciones tipológicas con motivos de las estaciones conocidas, se establece un horizonte cronológico que las relaciona con los yacimientos de El Cogul (Lleida) y Alpera (Albacete) en un primer momento - recordemos que se establecía entonces una cronologia paleolítica para estos lugares, aunque ya se comenzaba a considerar, por parte de estos autores, una posible atribución postpaleolítica-, pudiendo ser una de las escenas anterior, y que se desarrollaria hasta el Eneolítico o incluso más tarde. 


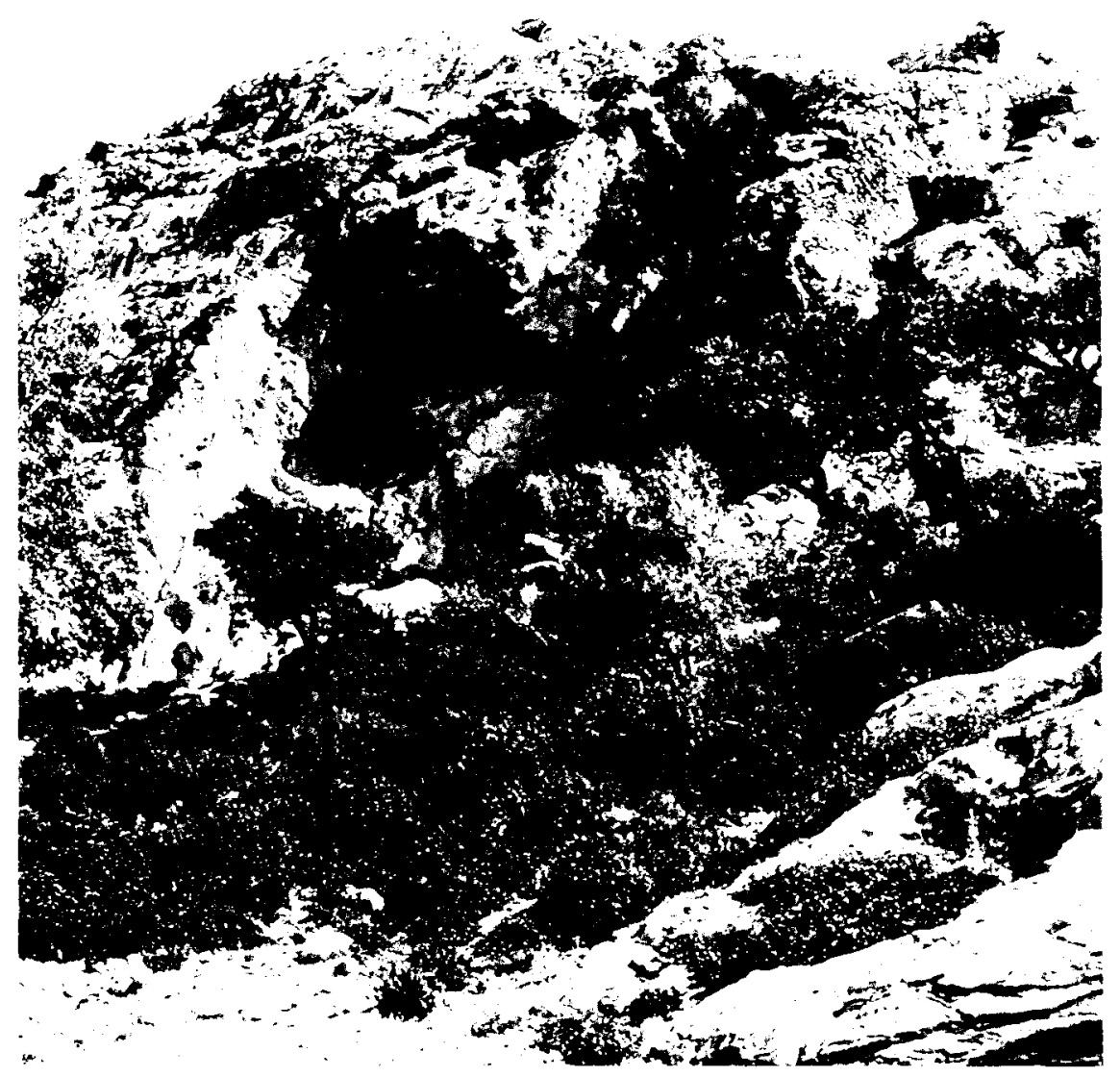

Fig. 1. Vista de conjunto de la Cueva del Tajo de las Figuras (Benalup, Cádiz).

H. Breuil, M. C. Burkitt y W. Verner, después de un detallado análisis de los distintos estilos, cuya diversidad hace suponer que corresponden a diferentes épocas, y de las superposiciones, establecen, dentro de esta cavidad, y en función del color, siete series. Determinan la existencia de quinientas siete representaciones, predominando las aves, seguidas de los cérvidos y las figuras humanas. Para ellos las pinturas blancas, extremadamente esquemáticas, serian las más antiguas y es evidente una datación neolítica, aunque ciertos motivos parecen anteriores, o posteriores, pues sus paralelos con representaciones cerámicas inducen a pensar en un horizonte cultural relacionado con la Edad del Cobre. 
Los sesenta fueron años de intensas revisiones. En este sentido citaremos, eludiendo el gran número de obras generales que se refieren al tema, las opiniones de E. Ripoll y P. Acosta al respecto. Para el primero (Ripoll Perello, E., 1968), quien opina que en el arte esquemáti$\mathrm{co}$, en grado mucho mayor que en el levantino, se dan lo que podríamos llamar estilos regionales que pueden corresponder a variantes culturales y también a desfases cronológicos, las tres fases establecidas en la Cueva del Tajo de las Figuras por J. Cabré y E. Hernández-Pacheco serían válidas, pero no su atribución cronológica, cuya datación difícilmente se remontaría, para la primera, más allá del 2500 a. C., pudiendo llegar, en las dos últimas, hasta finales de la Edad del Bronce. Acosta, al referirse a la zona gaditana, opina que «... constituye un caso aparte. Con el resto de las zonas españolas sólo tiene en común la esquematización, temas y tipología de varios motivos, pero el aspecto de sus pinturas presenta ciertas diferencias" (Acosta MARTinez, P., 1968:182). Recientemente esta autora ha reafirmado la complejidad de estas manifestaciones considerando que su origen podria explicarse hipotéticamente en relación al Neolítico meridional no cardial, aunque en los yacimientos arqueológicos de Andalucia no han aparecido, de momento, motivos figurativos entre los materiales atribuibles a este período (AcOsta MARTinez, P., 1984).

Desde 1988 y dentro del proyecto de investigación arqueológica Las manifestaciones rupestres prehistóricas de la zona gaditana se está procediendo a una nueva reproducción y estudio directo de la Cueva del Tajo de las Figuras y los restantes yacimientos, más de cien, con manifestaciones rupestres localizables en las sierras que bordean la antigua laguna de la Janda por el este y áreas próximas algo más alejadas, que esperamos nos permita aproximarnos al proceso evolutivo de estas representaciones y establecer su cronologia relativa. En este sentido podemos decir que los motivos pintados en estas estaciones, y especialmente en la Cueva del Tajo de las Figuras, se caracterizan por presentar, junto con determinadas tipologias relacionables con el denominado «fenómeno esquemático", unas fases con representaciones de tendencia naturalista que se diferencia de las consideradas esquemáticas y otras (“levantinas") que no pueden paralelizarse con las que se localizan aqui, con aspectos originales, muy poco frecuentes dentro del contexto del arte postpaleolítico de la Península Ibérica, como la existencia de representaciones de aves o la utilización de pintura blanca para plasmar abstracciones. Es por lo tanto aún prematuro establecer el horizonte cronológico de estas representaciones artisticas, aunque estarian claramente englobadas en momentos postpaleolíticos. 
Respecto a los grabados cabe decir que se localizan en el techo, fondo y pared izquierda, según se entra en la cavidad, y, como ya se ha mencionado, destacan entre trazos de época más reciente y estrias provocadas seguramente por haberse quitado los nidos de avispas terreras (Sceliphron sp) que aparecen en gran parte de las superficies, acciones antrópicas que parecen tener cierta antigüedad. A pesar de los numerosos investigadores que han estudiado o visitado la Cueva del Tajo de las Figuras, no hay ninguna referencia en la literatura científica a estos grabados, que convierten a este importante yacimiento en único y abren nuevas perspectivas a su lectura (Mas Cornella, M., y TORRa Colell, G., 1990).

En esta nota preliminar queremos presentar tres representaciones claramente figurativas y otras a las que por el momento no podemos dar una explicación plausible.

El encuadre cronológico paleolítico queda perfectamente confirmado, ya que estos grabados se encuentran infrapuestos a toda la secuencia pictográfica postpaleolítica, lo cual autentifica su antigüedad.

A continuación describimos detalladamente las figuras, entre las cuales destacan una cabeza de cierva, un protomos de caballo y una cabeza de cáprido.

\section{DESCRIPCIÓN DE LAS FIGURAS}

La primera de las representaciones es una cabeza de cierva (figs. 2 y 3), orientada a la izquierda, de gran tamaño (62 cm de largo por 37 de ancho), cuyo grabado, de sección en $V$ en casi todo el contorno, tiene una profundidad de $1,5 \mathrm{~mm}$ y una anchura que varia, desde $1,5 \mathrm{~cm}$, cuando aprovecha la roca-base, hasta $2 \mathrm{~mm}$ en el resto. Se observa claramente la parte correspondiente al morro, que se dibuja aprovechando un resalte natural de la roca, al igual que la oreja, la cual está insinuada en un trazo ascendente que se pierde debajo de uno de los abundantes restos de nidos de avispas terreras. Las parte inferior del cuello se prolonga en un trazo profundo y seguro hacia lo que seria el pecho.

En la zona correspondiente a la testuz, podemos apreciar un desconchón natural, que sin embargo no resta uniformidad a la figura. En el interior de la parte del morro, apreciamos, en un grabado ligeramente 
más fino, una silueta acuñada, y un trazo, que hemos interpretado como la oreja enhiesta de otro animal que se situaría en la zona inferior, al igual que otro trazo subcircular que aparece a unos $20 \mathrm{~cm}$ más abajo y que por su parte interpretamos como un ojo un tanto tosco. En la parte final del cuello se superpone una figura de cuadrúpedo $(12 \times 7 \mathrm{~cm})$ de tendencia esquemática, pintado a tinta plana en rojo.

La segunda de las figuras resulta algo más nítida, dada su posición en un plano subvertical. Es la que hemos denominado e identificado como un protomos de caballo que mira a la derecha (figs. 4 y 5). Tiene unas dimensiones de $40 \mathrm{~cm}$ de largo por 28 de ancho. La línea correspondiente al dorso tiene una profundidad de $2-3 \mathrm{~mm}$ y una anchura de 3-4. Se trata de un surco en $V$ que en la zona más alejada de la cabeza, es decir el inicio del dorso, se convierte en $U$ y tiene una anchura de $6 \mathrm{~mm}$, mientras que en la cabeza propiamente dicha la profundidad del trazo es ligeramente menor, así como menos ancha.

Falta la línea del pecho, posiblemente perdida por erosión, ya que coincide con el ángulo de entrada. Los trazos más evidentes son los que conforman lo que hemos identificado como la crin. La cabeza queda más o menos oculta por nidos de avispas terreras, y se hace más patente en la parte correspondiente a la testuz, que es de color oscuro debido a que en esta zona estuvo localizado un panal de abejas.

El inicio del morro y el ollar se dibujan aprovechando un resalte natural de la roca, perdiéndose casi por completo el trazo, que en esta zona adquiere su máxima finura. A continuación aparece la línea correspondiente a la quijada, la cual deberia unirse al pecho, que no existe.

La crin sobresale $2 \mathrm{~cm}$ del inicio de la cabeza y posee un trazo perpendicular que podría interpretarse como la oreja. Al igual que en la figura anterior, en la parte del inicio del dorso, apreciamos un cuadrúpedo acéfalo $(15 \times 14 \mathrm{~cm})$ de tendencia esquemática, pintado a tinta plana en rojo, también superpuesto al grabado.

La tercera de las representaciones es la que quizás ofrece menos dudas en cuanto a su atribución, a pesar de las dificultades existentes para verla dada la escasísima profundidad del trazo. Se trata de una cabeza de cáprido (figs. 6 y 7), mirando hacia la izquierda, con unas dimensiones de $19 \mathrm{~cm}$ de largo por 9 de ancho. Como ya hemos mencionado, está realizado con un surco muy somero en forma de $U$ de unos $2 \mathrm{~mm}$ de anchura y una profundidad que en muchos casos no supera el medio milímetro. Su posición es ligeramente rampante, como si estuviese en actitud de bramar. Domina, por la firmeza del trazo, el largo cuerno de casi $13 \mathrm{~cm}$ de longitud. Tiene una oreja que se sitúa prácticamente 


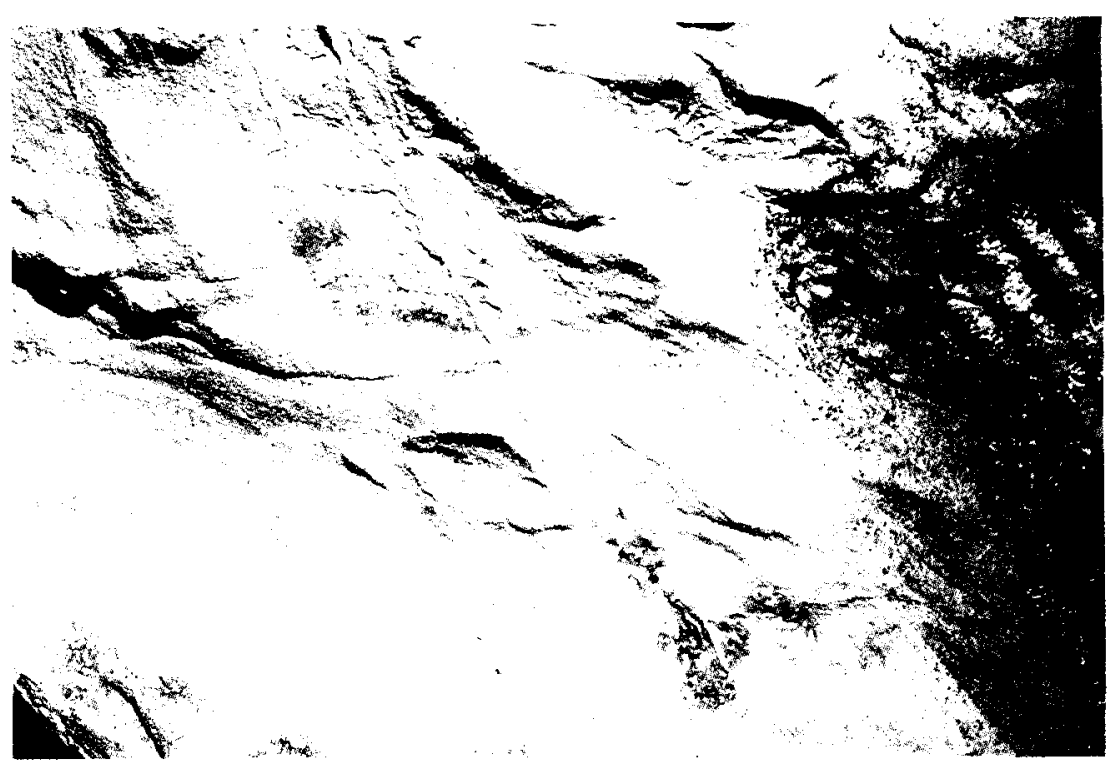

Fig. 2. Fotografia de la cabeza de cierva descrita en primer lugar.

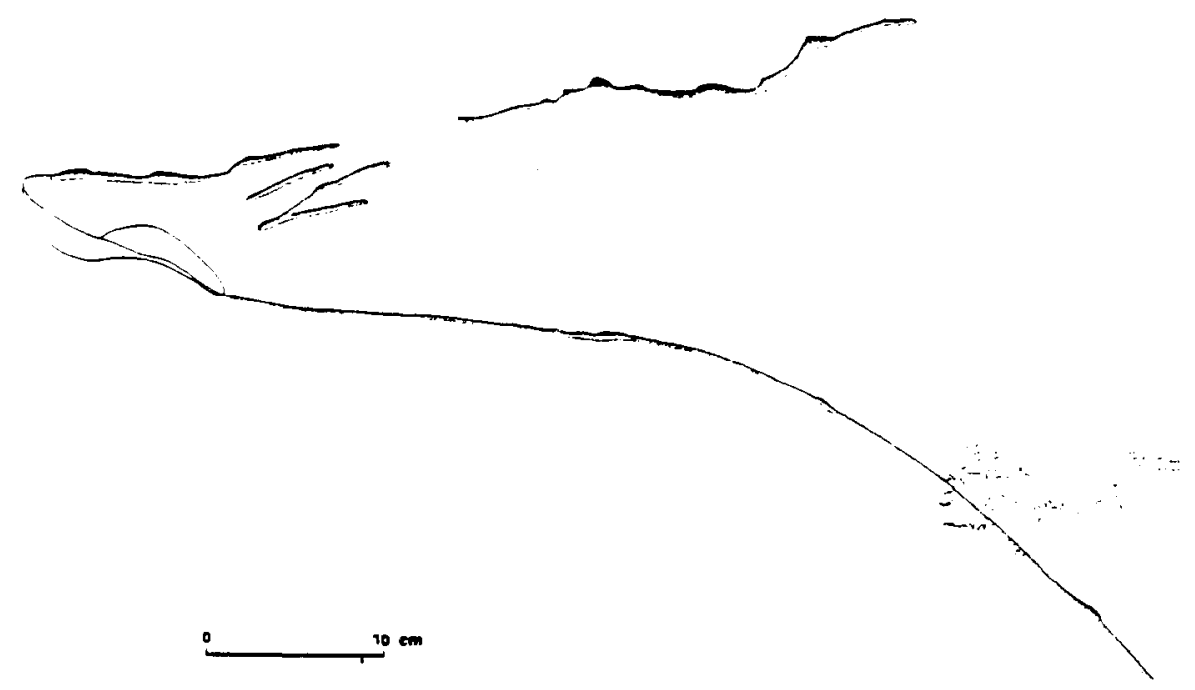

Fig. 3. Calco-reproducción de la cabeza de cierva. En la parte inferior del cuello puede apreciarse un cuadrúpedo superpuesto, pintado. 


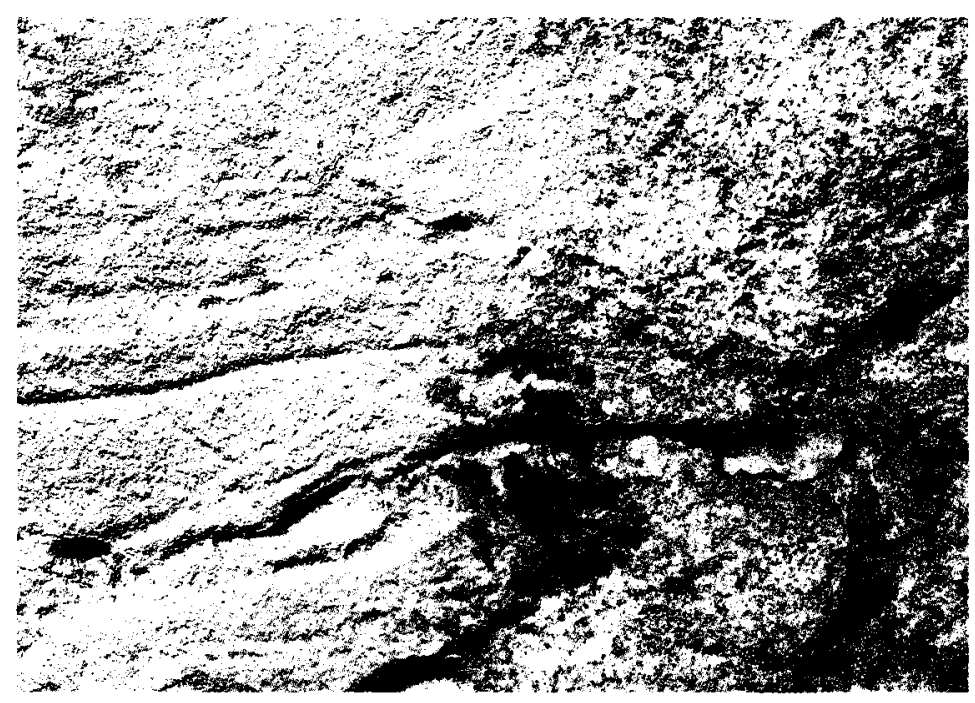

Fig. 4. Detalle de la parte anterior del protomos de caballo.

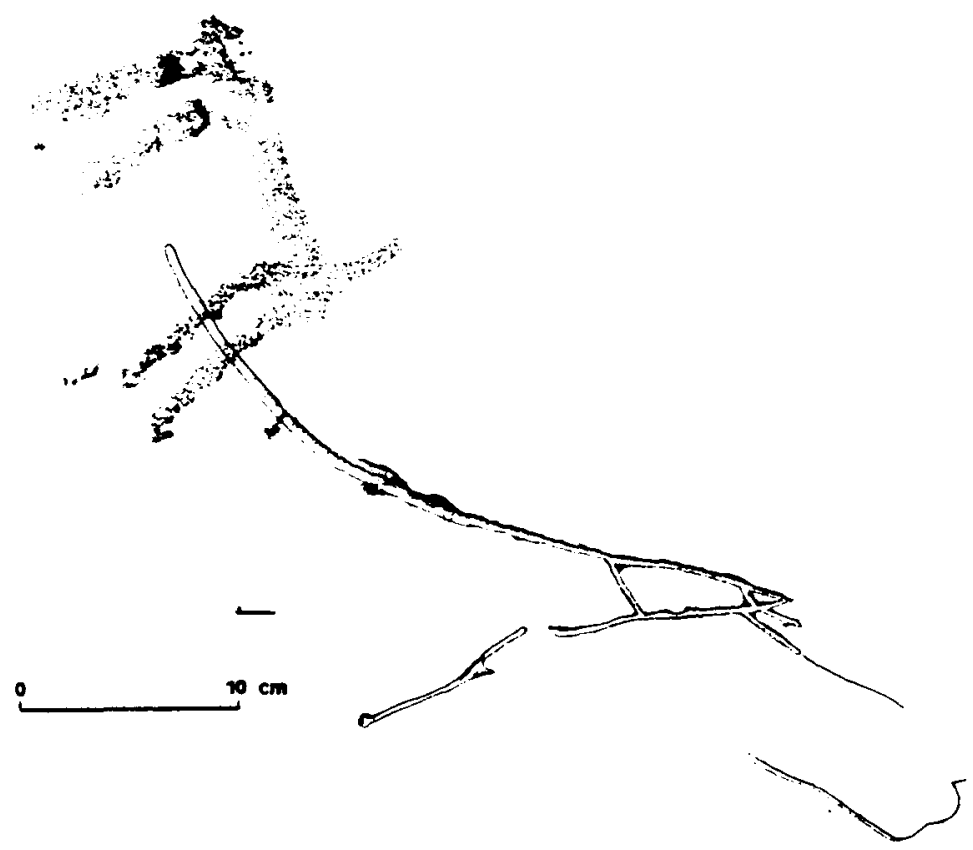

Fig. 5. Calco-reproducción del protomos de caballo. Se observa la superposición pintada de un cuadrúpedo acéfalo en el inicio del dorso. 


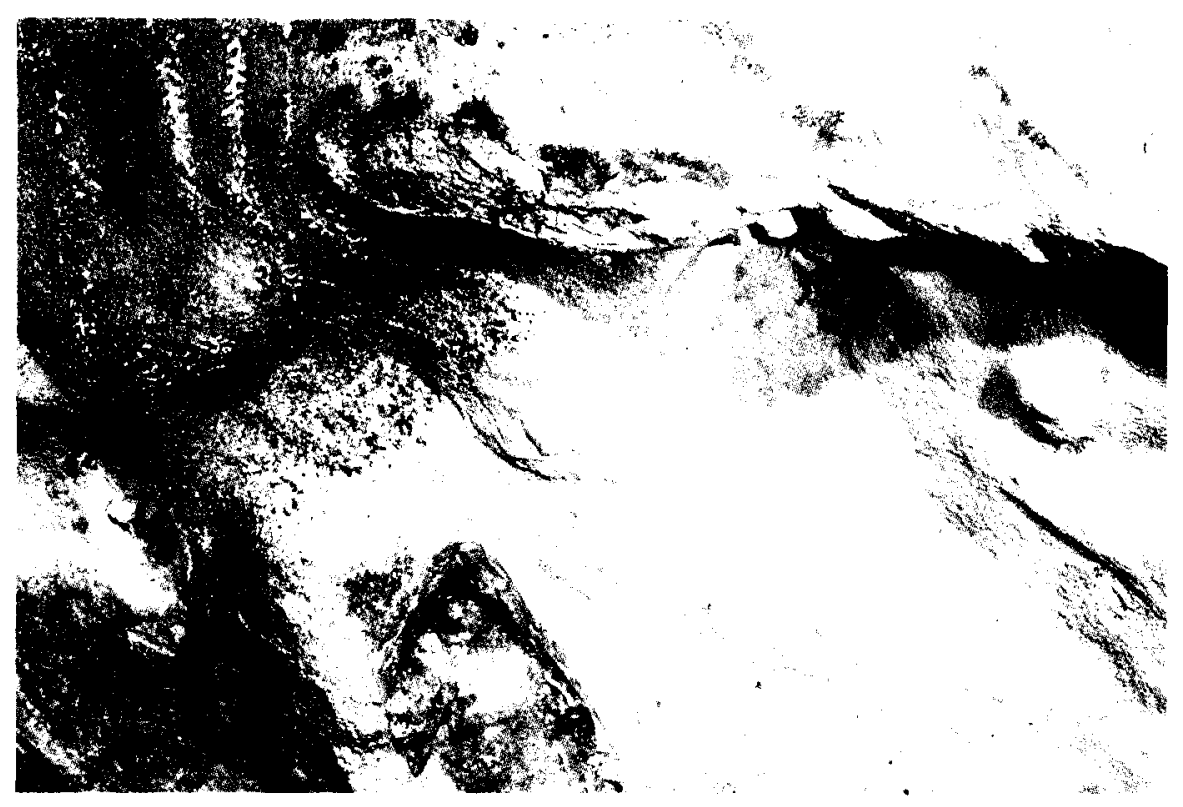

Fig. 6. Fotografia de la cabeza de cáprido.
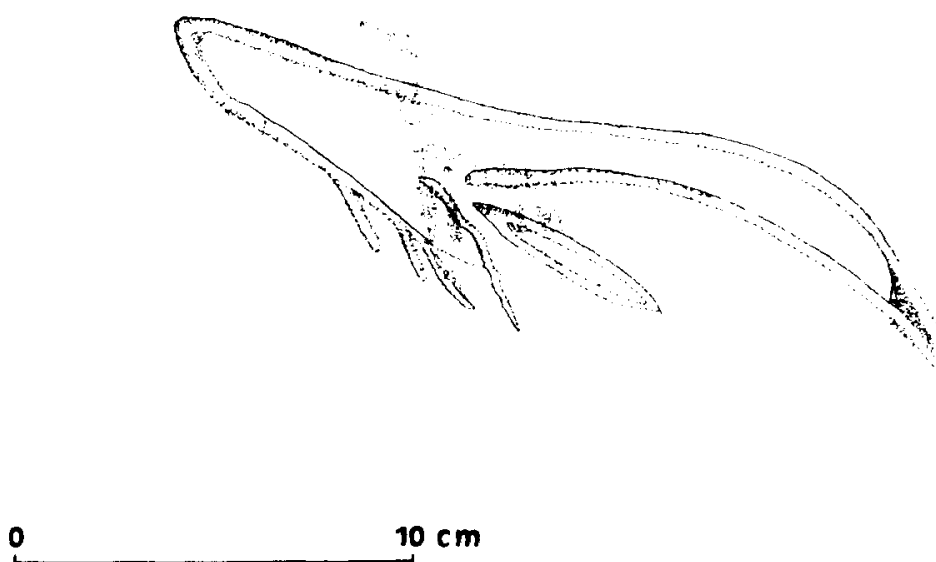

Fig. 7. Calco-reproducción de la cabeza de cáprido, claramente infrapuesta a un cuadrúpedo pintado. 
paralela al cuerno, con una longitud de $5 \mathrm{~cm}$. En la parte inferior de la quijada se aprecian cuatro trazos a modo de barba. El conjunto de la cabeza, excepto el cuerno, posee una menor nitidez, ya que el panal que cubría al caballo antes descrito, también afecta parcialmente a esta figura y provoca un ligero cambio en la coloración del surco.

Al igual que a las otras figuras, a ésta se le superpone claramente un cuadrúpedo $(7 \times 8 \mathrm{~cm})$ de tendencia esquemática, pintado a tinta plana en rojo. A unos centímetros a la izquierda del morro de esta figura hemos distinguido otras líneas de sección en $U$ con una profundidad de 1-2 mm y 1-2 de ancho, que no forman ninguna representación clara.

En las paredes de la Cueva del Tajo de las Figuras existe un gran número de surcos, en algunos casos líneas de una longitud considerable, que en un principio no parecen dibujar ningún motivo figurativo, aunque se infraponen siempre a toda la secuencia pictórica postpaleolitica, y que debido a las condiciones en que se han realizado estos hallazgos y el escaso tiempo disponible no han podido ser estudiados detenidamente. Esperamos poder continuar y finalizar nuestras investigaciones documentando estos motivos de forma completa en una futura campaña.

La última representación que configura este avance es un largo trazo de más de 2 metros que ocupa prácticamente toda la bóveda del covacho. El surco es bastante ancho (entre 3 y $9 \mathrm{~mm}$ ) y profundo $(2-5 \mathrm{~mm}$ ) con una sección en $U$ bastante cerrada y en algunas zonas en $V$. En el sector oriental se aprecia una forma subtriangular compuesta. En la otra extremidad (occidental), sin conexión con la línea del techo, existe un surco de trazado sinuoso. No insistimos en el análisis de estas composiciones ya que quedan pendientes de un examen posterior más detallado.

\section{CONSIDERACIONES GENERALES}

Cuando se revisa el repertorio bibliográfico en busca de paralelos, surge de forma espontánea relacionar estos grabados con la riquisima colección de plaquetas halladas por L. Pericot en la cueva de Parpalló (Gandía, Valencia) y en general con la tradición iconográfica del Sur de la Península Ibérica.

El protomos de caballo posee sus paralelos más claros e inmediatos en dos plaquetas halladas en el nivel atribuido por L. Pericot al Solutrense Medio (fig. 8, I-II) con el mismo tipo de despiece de la crin que sobre- 

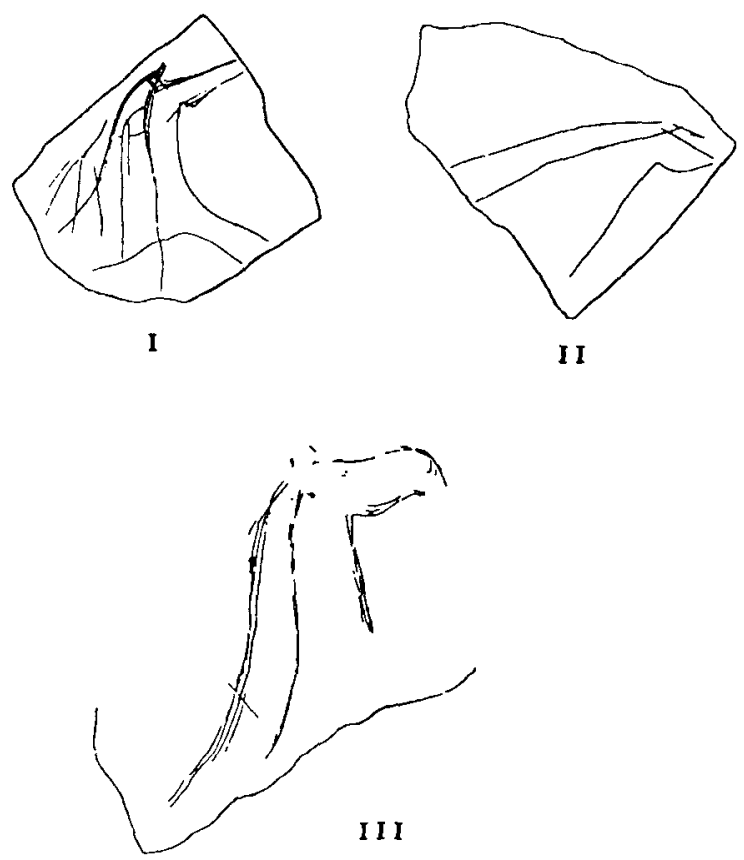

Fig. 8. Paralelos para el protomos de caballo, procedentes de la cueva del Parpalló. Los dos primeros están encuadrados en el Solutrense Medio y el tercero en el Solutreo-Auriñaciense Final. (Según L. Pericot).

sale a la cabeza propiamente dicha (PERICOT, L., 1942). Esta "convención», más depurada, se aprecia en otra plaqueta encuadrada en el «Solutreo-Auriñaciense Final» (fig. 8, III), es decir en el momento que nosotros denominamos como Solutrense Superior Evolucionado y que J. M. Fullola (1979) califica como Solútreo-Gravetiense.

Conviene destacar que en el motivo de la Cueva del Tajo de las Figuras no se aprecia la convención estilística del morro "pico de pato", tan caracteristica del Solutrense.

A pesar de la relativa proximidad de la cueva de la Pileta (Benaoján, Málaga), las características estilísticas de esta estación son bastante diferentes, estando más en sintonia con nuestros grabados la tradición pictórica observada en la cueva de Doña Trinidad de Ardales (Málaga) (BREUIL, H., 1921). 
Es precisamente en esta cueva (fig. 9, VII-VIII) donde también se encuentran los primeros paralelos de la cabeza de cierva. Tanto las figuras de ciervas grabadas como las pintadas en amarillo poseen unas cabezas muy estilizadas y alargadas — similares a la del Tajo de las Figuras - y se encuadran culturalmente en un Solutrense Medio-Final o Magdaleniense Inicial (EsPejo, M. ${ }^{a}$ J., y Cantalejo, P., 1988).

Como en el caso del équido, tenemos que recurrir nuevamente a Parpalló para encontrar los paralelos más próximos. Se trata de tres plaquetas grabadas pertenecientes a las tres fases culturales del Solutrense (fig. 9, IV, V, VI), en las que se aprecia en todos los ejemplos una cabeza de cierva muy estilizada y alargada. La similitud más clara es con la representación del Solutrense Inferior, aunque deberiamos prescindir de los trazos de las orejas y el dorso. En la plaqueta correspondiente al Solutrense Medio - que aqui aparece invertida de derecha a izquierda para que se vea en el mismo sentido que nuestra figura-, se observa el mismo alargamiento y proyección hacia adelante del cuello y la cabeza, construidos con tres líneas elementales, en las dos ciervas que se hallan superpuestas.

Para la tercera de las figuras, el cáprido, los paralelos no son tan evidentes y por el momento se hallan sólo en Parpalló (fig. 9, I-II). En este caso, también se encuadran en el Solutrense, aunque las representaciones sean más elaboradas que la figura que nos ocupa. Sin embargo, todas ellas poseen un morro más o menos redondeado y un largo cuerno que se prolonga hacia atrás. Quizás la pieza más próxima sea la atribuida al Solutrense Inferior (fig. 9, III), aunque también posee algunos rasgos de la número 2 que fue hallada sin estratigrafia.

Los paralelos para las últimas figuras aqui descritas son inexistentes por el momento y las hemos dejado en suspenso con el fin de estudiarlas con más detalle en futuros trabajos.

Como rasgo general se observa un soporte erosionado o "frotado" con posterioridad a la ejecución del grabado y que, por tanto, afecta a la integridad de las figuras. Este hecho hace que, a veces, la identificación zootécnica se vea dificultada por la ausencia de ciertos rasgos anatómicos y que algunos de los trazos inconexos, pertenecientes sin duda a otras figuras, no puedan ser interpretados en toda su extensión.

El tipo de trazo más frecuente es el simple único, aunque existen algunos desdoblamientos de líneas en determinados puntos, sin duda producidos por los propios accidentes naturales del soporte. No hemos podido constatar de forma evidente la existencia de algún tipo de modelado, sin embargo, algunos detalles de ciertas representaciones nos po- 

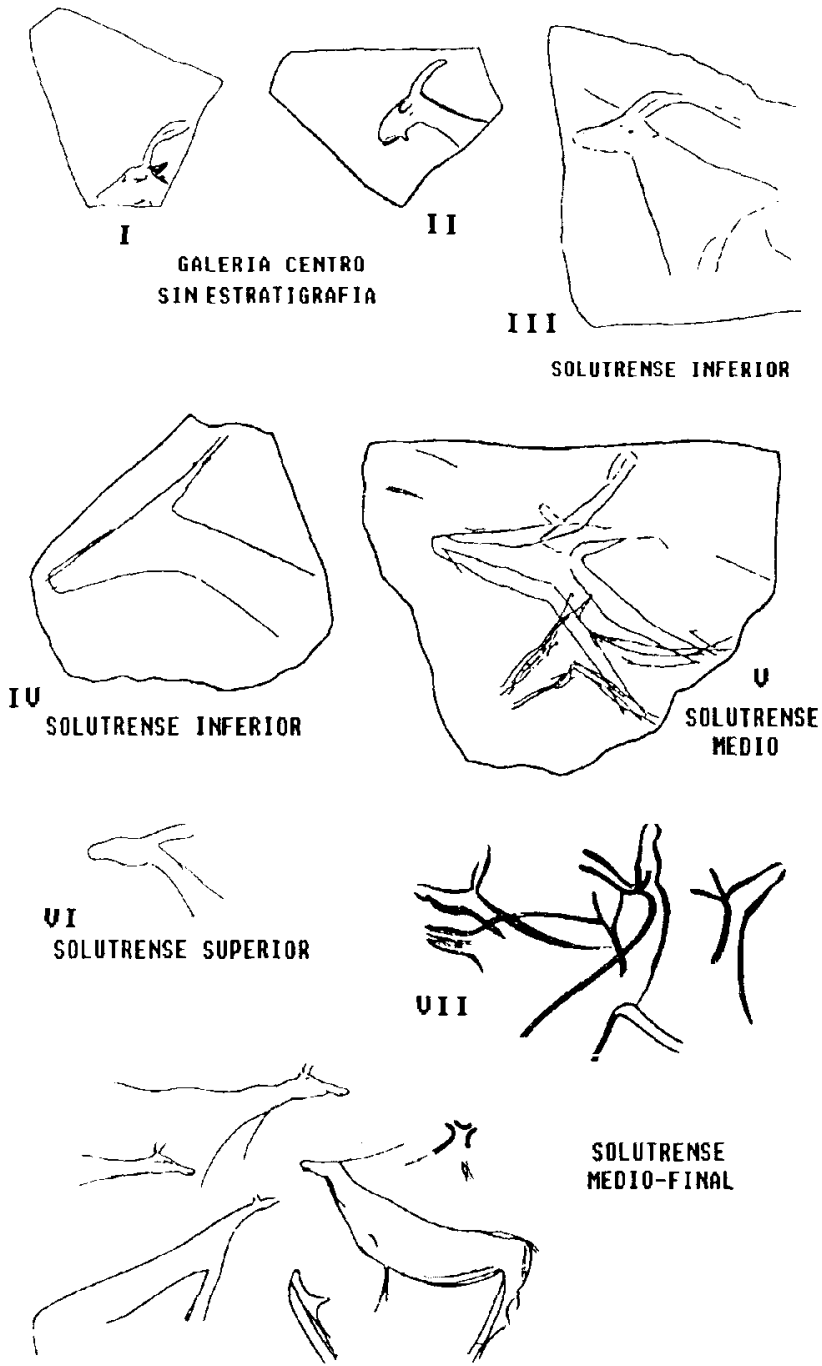

SOLUTRENSE MEDIO-F INAL

\section{UI I I}

Fig. 9. Paralelos correspondientes a las dos restantes figuras descritas en este artículo. Las seis primeras plaquetas proceden de la cueva del Parpalló (según L. Pericot), y las dos últimas - representaciones parietales - son de la cueva de Doña Trinidad de Ardales (según H. Breuil). Los numeros I y II fueron hallados en la Galería centro, sin estratigrafía y el III se encuadra en el Solutrense Inferior. Los siguientes números IV, V y VI se encuadran en el Solutrense Inferior, Solutrense Medio y Solutrense Superior respectivamente. 
drían inducir a pensar en la existencia del mismo. Así, observamos que en la primera figura, la cabeza de cierva, se aprovecha una grieta de la roca para resaltar la oreja que no aparece explícitamente marcada y el morro se dibuja a partir de un resalte. En el caso del équido sucede algo parecido, ya que el morro aprovecha una forma redondeada, obviando el trazo.

La sencillez esquemática es la característica más generalizada del conjunto que aqui presentamos. Las figuras se reducen a simples siluetas, sin rellenos, a excepción de la crin del équido.

Según J. Fortea (1978) la convención trilineal se desarrolla a lo largo de todo el horizonte Solutrense y posee la misma especial idiosincrasia que las industrias halladas para este período en la zona andaluza.

Como hemos podido apreciar, casi todos los paralelos estilísticos nos llevan a encuadrar las representaciones paleolíticas inéditas de la Cueva del Tajo de las Figuras en un momento Solutrense sensu lato. Esperamos que las futuras investigaciones que se están llevando a cabo en el marco del proyecto de investigación arqueológica Las manifestaciones rupestres prehistoricas de la zona gaditana, nos permitan hallar, identificar y describir nuevas representaciones que nos conduzcan a completar de una forma más precisa esta "provincia pictórica mediterránea" (GRAZIOSI, P., 1968).

El hallazgo de figuras superopaleoliticas en esta estación, que posee tal profusión de pinturas postpaleolíticas, nos confirma que se trata de un lugar con una importantísima posición estratégica, al borde de la antigua laguna de la Janda, por cuyos alrededores debieron de transitar tanto hombres como animales desde la más remota Prehistoria hasta nuestros dias. 


\section{BIBLIOGRAFIA}

Acosta Martínez, P., 1968, La pintura rupestre esquemática en España. Salamanca, Universidad de Salamanca, 250 págs.

Acosta Martinez, P., 1984, «El arte rupestre esquemático ibérico: problemas de cronologia preliminares", (FORTEA, J., ed.): Scripta Praehistorica Francisco Jordá Oblata. Salamanca, Ediciones Universidad de Salamanca, págs. 31-61.

BreuIL, H., 1929, "Nouvelles cavernes ornées Paléolithiques dans la province de Málaga", L'Anthropologie, t. XXXI, págs. 239-253, 11 figuras.

BreuIL, H., y BuRkitt, M. C., 1929, Rock paintings of southern Andalusia. A description of a Neolithic and Copper Age art group. Oxford, Clarendon Press, 88 págs.

Cabre, J., y Hernandez-PACheco, E., 1914, "Avance al estudio de las pinturas prehistóricas del extremo sur de España (Laguna de la Janda)", Trabajos de la Comisión de Investigaciones Paleontológicas y Prehistóricas, 3. Madrid, 35 págs.

Espejo, M. ${ }^{\text {a }}$ del Mar, y Cantalejo, P., 1988, "Cueva de Ardales, yacimiento recuperado", Revista de Arqueología, núm. 85, págs. 14-24.

Fortea Pérez, F. J., 1978, "Arte Paleolítico del Mediterráneo español», Trabajos de Prehistoria, vol. 35, págs. 99-149, 25 figuras.

Fullola Pericot, J. M.a, 1979, "Las industrias del Paleolítico Superior en el Mediterráneo español», S.I.P. Trabajos Varios, núm. 60. Valencia, 262 págs.

Grazıosı, P., 1968, “L'Art paléo-épipaléolithique de la Province Méditerranéenne et ses nouveaux documents d'Afrique du Nord et du Proche Orient", (RIPOLl Perello, E., edit.) Simposio Internacional de Arte Rupestre, Barcelona 1966. Diputación Provincial de Barcelona, págs. 265-271. 
Hernandez-Pacheco, E., y Cabre, J., 1913, "La depresión del Barbate y sus estaciones prehistóricas», Boletín de la Real Sociedad Española de Historia Natural, XIII, págs. 349-359.

Mas Cornella, M., y Torra Colell, G., 1990, "Arte rupestre en Cádiz, documentación e investigación", Revista de Arqueologia, núm. 113, págs. 14-22.

MolinA, V., 1913, “Arqueología y prehistoria de la provincia de Cádiz en Lebrija y Medinasidonia», Boletín de la Real Academia de la Historia. LXII, págs. 554-562.

Pericot, L., 1942, La Cueva del Parpalló (Gandia). Excavaciones de Servicio de Investigación Prehistórica de la Diputación de Valencia. Madrid, C.S.I.C. Instituto Diego Velázquez, 351 págs.

Ripoll Perello, E., 1968, "Cuestiones en torno a la cronología del arte rupestre postpaleolítico en la Península lbérica", (RIPOLL PERELló, E., ed.): Simposio Internacional de Arte Ruprestre. Barcelona, 1966. Diputación Provincial de Barcelona, págs. 165-192. 